AsClepIo. Revista de Historia de la Medicina y de la Ciencia

65 (2), julio-diciembre 2013, p017

ISSN-L:0210-4466

http://dx.doi.org/10.3989/asclepio.2013.17

\title{
LA CONSTRUCCIÓN DEL SUJETO PELIGROSO EN ESPAÑA (1880-1936). EL PAPEL DE LA PSIQUIATRÍA Y LA CRIMINOLOGÍA ${ }^{1}$
}

\author{
Ricardo Campos \\ Instituto de Historia, Centro de Ciencias Humanas y Sociales (CSIC) \\ ricardo.campos@cchs.csic.es
}

Recibido: 20 noviembre 2012; Aceptado: 20 mayo 2013.

Cómo citar este artículo/Citation: Campos, Ricardo (2013), "La construcción del sujeto peligroso en España (1880-1936). El papel de la psiquiatría y la criminología", Asclepio 65 (2): p017. doi: http://dx.doi.org/10.3989/asclepio.2013.17

RESUMEN: El objetivo de este trabajo es analizar cómo la psiquiatría y la criminología contribuyeron a patologizar al criminal y a criminalizar la locura, ahondando en la estigmatización y marginación del enfermo mental y los individuos con comportamientos desviados. Partiendo de las reflexiones de Michel Foucault sobre la noción de individuo peligroso y anormal, pretendo enmarcar la construcción del sujeto peligroso en España durante el periodo 1880-1936 tomando en cuenta tres cuestiones: 1) el impacto del degeneracionismo en los peritajes psiquiátricos y la recepción de la antropología criminal en España durante las décadas de 1880 y $1890 ; 2$ ) la pugna entre psiquiatras y juristas en torno a los conceptos de responsabilidad, libre albedrío y peligrosidad potencial, que condujo a un sector de la psiquiatría a introducir a mediados de la década de 1890 una noción, la de responsabilidad atenuada, que permitía una nueva definición del individuo peligroso y la defensa social; y 3) el tratamiento de la peligrosidad social del enfermo mental por parte del movimiento de higiene mental entre 1920 y 1936.

PALABRAS CLAVE: Psiquiatría; criminología; peligrosidad; defensa social; higiene mental; España; siglos XIX y XX.

\section{THE CONSTRUCTION OF THE DANGEROUS SUBJECT IN SPAIN (1880-1936). THE ROLE OF PSYCHIATRY AND CRIMINOLOGY}

ABSTRACT: The objective of this work is to examine how psychiatry and criminology contributed to the pathologisation of criminals and the criminalisation of madness, thus increasing the stigmatisation and marginalisation of mental patients and deviant individuals. Based on Michel Foucault's ideas on the dangerous and abnormal individual, I intend to describe the construction of the dangerous subject in Spain between 1880 and 1936 taking into account three main issues: 1) the influence of degenerationism on psychiatric court reports and the reception of criminal anthropology in Spain during the decades of the 1880s and 1890s; 2) the conflict between psychiatrists and law concerning the concepts of responsibility, free will and potential risk, showing how around the mid 1890s a sector of the psychiatric profession introduced a notion, that of diminished responsibility, that fostered a new perception of the dangerous individual and a redefinition of social defence; 3 ) the treatment of the social dangerousness of the mentally ill by the mental hygiene movement from 1920 to 1936.

KEY WORDS: Psychiatry; criminology; dangerousness; social defense; mental hygiene; Spain; nineteenth and twentieth centuries.

Copyright: (c) 2013 CSIC. Este es un artículo de acceso abierto distribuido bajo los términos de la licencia Creative Commons Attribution-Non Commercial (by-nc) Spain 3.0. 


\section{INTRODUCCIÓN}

Existe un amplio consenso historiográfico en convenir que la patologización del crimen fue un proceso vinculado al nacimiento y desarrollo de la psiquiatría. Los interrogantes historiográficos que suscita la vinculación entre crimen y locura son numerosos y forman parte de innumerables trabajos. Buena parte de la producción se ha centrado en explicar cómo se produjo la identificación entre crimen y locura, en analizar cuales fueron los discursos y prácticas que difuminaron las fronteras entre ambos fenómenos y en estudiar cómo y por qué se pasó de propugnar la curación de todos los enfermos mentales, incluidos los que cometían actos criminales, a plantear su castigo.

Michel Foucault, cuya influencia en este terreno es indiscutible, planteó que, desde sus inicios, la psiquiatría codificó la locura como enfermedad y como peligro, y que los momentos "fuertes» de su historia se habrían producido "cuando las dos codificaciones» habían "estado efectivamente ajustadas» o cuando había existido "un único tipo de discurso, un único tipo de análisis, un único cuerpo de conceptos» que habían permitido «constituir la locura como enfermedad y percibirla como peligro» (Foucault, 2001, 112). Este planteamiento, defendido por el autor francés en diversos escritos (Foucault, 1990a; Foucault, 1990b), ha servido para desarrollar una prolífica línea de trabajo que considera a la psiquiatría como una rama de la higiene pública y al asilo como una institución de exclusión, control y defensa social (Dörner, 1974; Scull, 1979; Castel, 1980; Álvarez-Uría, 1983). Esta perspectiva tendría como trasfondo un interés principal -el estudio de las técnicas de disciplinamiento y normalización de la población- y llevaría implícita la negación del carácter científico de la psiquiatría.

Sin embargo, algunos de estos puntos han sido matizados y discutidos. Así, Gladys Swain defendió que, en los orígenes del alienismo, en el proyecto de tratamiento moral de Pinel, no hubo un proyecto represivo y de exclusión de la locura, sino un proyecto positivo que buscaba el tratamiento médico y el alivio del sufrimiento del enfermo (Swain, 1977). Por su parte, Lantéri-Laura también consideraba que el «paradigma de la alienación mental», representado por las obras de Pinel y Esquirol, no entrañaba estrictamente un proyecto de defensa social, pues frente a los actos criminales cometidos por el sujeto alienado prevalecía su estatuto de enfermo (Lantéri-Laura, 2000, 73-133).

Es cierto que, desde mediados del siglo XIX, con la adopción del degeneracionismo como paradigma de la psiquiatría, la identificación entre locura y crimen se hizo más evidente, resultando difícil marcar las diferencias entre ambos. La irrupción de las tesis de la Antropología criminal contribuyó a borrar dichas fronteras, dejando una huella que perduró más allá de la vigencia de sus propias teorías (Peset, 1983). En este sentido, una cuestión fundamental que marcó todos los modelos médicos de criminalidad fue la preocupación de la psiquiatría por la existencia de las «locuras parciales». Estas, en sus distintas formas, habrían jugado un papel esencial tanto en la vinculación entre locura y crimen como en la propia definición psiquiátrica de la peligrosidad (Huertas, 2011).

Ahora bien, que exista un continuum histórico no significa, desde nuestro punto de vista, que esta configuración responda a ningún orden, ni que las etapas en la definición de la peligrosidad y la vinculación entre locura y crimen se superpongan teleológicamente. Las diferencias de discursos y propuestas de los psiquiatras españoles en las décadas de 1880 y 1930 ejemplifican bien este extremo. Entre ambos periodos existe un vínculo encarnado por la existencia de las locuras parciales, pero la visión del sujeto peligroso y del trato que debía recibir eran distintas. Los psiquiatras degeneracionistas de la década de 1880 introdujeron el debate sobre el libre albedrío y la irresponsabilidad de los enfermos mentales que cometían actos contra la sociedad. No parece que desarrollaran, aunque la criminología sí lo estuviera haciendo, un discurso sobre la defensa social o que concibieran, más allá de la retórica, a la psiquiatría como una rama de la higiene pública. Sin embargo, los psiquiatras de la Il República, inmersos en el movimiento de higiene mental, se alinearon con la higiene, la defensa social y la profilaxis del crimen, desarrollando un discurso muy elaborado sobre la peligrosidad potencial del enfermo mental y la necesidad de implementar medidas de defensa social.

Por otra parte, los intereses profesionales y las estrategias de legitimación de la profesión también tuvieron un papel importante en la gestación de las vinculaciones entre locura, crimen y peligrosidad. La presencia de los psiquiatras en los tribunales de justicia como peritos fue fundamental en este sentido. Sus intereses en tanto que peritos no fueron exactamente los mismos que desarrollaron en el terreno clínico. El espacio en el que ejercían su profesión es un elemento de enorme importancia que contribuye a comprender mejor el desarrollo de la peligrosidad en el ámbito psiquiátrico (Goldstein, 1987; Campos, 1999; Huertas, 2002). Asimismo, es necesario establecer los puntos de conexión entre las propuestas psiquiátricas y las legislaciones -y su aplicación- para poder calibrar, más allá de las proclamas, la textura de las medidas que se aplicaban. La historiografía muestra, en este sentido, que no siempre las leyes se hicieron a imagen y semejanza de las reivindicaciones psiquiátricas (Renneville, 2003).

El objetivo del presente trabajo es analizar algunas de las vías a través de las que se construyó el concep- 
to de peligrosidad en España entre 1880 y 1936. Para ello, abordaré algunos aspectos relacionados con la psiquiatría y la criminología, centrándome en el papel de los peritajes psiquiátricos durante la década de 1880 como principal vía de penetración y reelaboración del degeneracionismo y el lombrosianismo, para, a continuación, tratar la influencia posterior de la doctrina de la responsabilidad atenuada en la definición de un nuevo concepto de peligrosidad y de sujeto peligroso vinculado a la defensa social. Por ultimo, estudiaré cómo los psiquiatras militantes en el movimiento de higiene mental de las décadas de 1920 y 1930 intentaron ofrecer una definición de la peligrosidad y de la prevención del delito más sofisticada, llegando a plasmarse durante la II República en leyes represivas como la Ley de Vagos y Maleantes.

\section{LA LOCURA EN LOS TRIBUNALES DE JUSTICIA}

En la década de 1880 la psiquiatría española adoptó el degeneracionismo como modelo explicativo del crimen, abandonando el de monomanía (Campos, Martínez, Huertas, 2000; Plumed, Rey, 2002). Su aceptación fue simultánea a la incorporación de las teorías lombrosianas, dando lugar a una amalgama de ideas en la que resultaba difícil distinguir lo que provenía específicamente de cada una de ellas (Campos, Huertas. 2013). El hecho de que la principal vía de penetración del degeneracionismo y la Antropología criminal fueran, al menos en un primer momento, los peritajes psiquiátricos llevados a cabo por José María Esquerdo y su círculo de colaboradores en un serie de casos criminales de gran impacto social, marcó una clara deriva del alienismo hacia la identificación del loco con el criminal (Álvarez-Uría, 1983; González González, 1994; Huertas, 2004; Campos, 2012). Ambas doctrinas tuvieron una importancia fundamental en la construcción de unas de las primeras imágenes del sujeto peligroso de la España decimonónica, superando la importancia que había tenido en un primer momento la monomanía (Huertas, Martínez-Pérez, 1993).

La década de 1880 fue crucial para la definición de la psiquiatría como ciencia. La convergencia de diversas transformaciones en los ámbitos científico, judicial, político y cultural hizo posible la configuración y visibilización definitiva del loco criminal (Maristany, 1973, 1998; Álvarez-Uría, 1983; Campos, 2012). A los cambios operados en el campo psiquiátrico hay que sumar los producidos en el campo jurídico, plasmados en el proceso de codificación penal y en la promulgación de leyes como la de enjuiciamiento criminal de septiembre de 1882, que jalonan la transición desde una justicia anclada en una cultura judicial histórica de corte inquisitorial a otra moderna y acorde con los principios liberales (Ríos-Font, 2005; Petit, 2005; Campos, 2012).
En este contexto, Esquerdo lideró una ofensiva en las salas de justicia con el propósito de dar a conocer a la opinión pública y a los magistrados los progresos del alienismo y legitimarlo socialmente como disciplina científica. La estrategia se basaba en demostrar públicamente la relación entre locura y crimen, reclamando para la psiquiatría al loco criminal (Álvarez-Uría, 1983). El inicio de la campaña lo marcaron los casos de Otero, que en 1879 atentó contra el Rey Alfonso XII (Consiglieri, Villasante, 2008) y el de José Garayo "el Sacamantecas», que, entre 18701879 , violó y destripó a varias mujeres en la provincia de Álava (Huertas, 2004). Esquerdo actuó como perito de la defensa en ambos casos, defendiendo la locura y por tanto la irresponsabilidad penal de los acusados, aunque sin éxito, pues ambos fueron condenados a muerte y ajusticiados.

Como respuesta a las decisiones judiciales, entre marzo de 1880 y febrero de 1881 Esquerdo dictó cuatro conferencias - bajo el título común de Locos que no lo parecen- consagradas a demostrar que los jueces habían cometido un error judicial en los dos casos casos². Estas conferencias muestran cómo se estaba produciendo el cambio de modelo explicativo del crimen desde la monomanía al degeneracionismo. Así, las dos primeras, dedicadas al caso Otero, estaban circunscritas al paradigma de la monomanía (Conseglieri, Villasante, 2008). Sin embargo, en las dos siguientes, centradas en "El Sacamantecas», se aprecia un interesante cambio, pues Esquerdo, pese a diagnosticarle una "monomanía genésica», utilizaba numerosos elementos del degeneracionismo y la Antropología criminal -como los estigmas físicos y la herencia biológica - para demostrar su enfermedad mental (Álvarez-Uría, 1983; Huertas, 2004). De este modo, la descripción antropométrica de «El Sacamantecas» resaltaba con fuerza sus estigmas físicos, que adquirían el valor de signo inequívoco de su naturaleza enferma y su locura:

Garayo, bajo el punto de vista somático, presenta una cabeza contrahecha, deforme; pero ique deformidad, señores! Ancha en su base, angosta en su bóveda, estrecha en la frente y espaciosa hacia el occipucio; la curvadura posterior está tan deprimida, que desde lo lato de la cabeza hasta la parte posterior de la cerviz se baja por un solo plano; solo a los lados y partes inferiores de dicho plano se distinguen dos anchas prominencias: el diámetro transversal predomina sobre el ántero-posterior, y de las dos mitades en que este la divide es la derecha mucho mayor que la izquierda: no presenta en toda su extensión más que una cicatriz de 3 centímetros de extensión, huella de una antigua herida (Esquerdo, 1881, 155).

La descripción de su cuerpo se completaba con la de su rostro, que se caracterizaba por tener los pómulos salientes, los ojos pequeños, hundidos, desiguales, desnivelados y las cejas pobladas, rasgos que le daban 
"un aspecto tenebroso" y una mirada "fiera e intensa» (Esquerdo, 1881, 156). Igualmente, Esquerdo establecía una estrecha vinculación entre su enfermedad mental y su herencia biológica, trazando un árbol genealógico familiar profundamente patológico en el que sus padres, hermanos, sobrinos e hijos padecían locura (Esquerdo, 1881, 159).

Desde ese momento, los integrantes de su círculo comenzaron a intervenir públicamente con el objeto de demostrar que muchos locos terminaban en prisión o en el patíbulo por no haberse realizado peritajes psiquiátricos o no haberse tomado en cuenta sus resultados cuando se practicaban. Paralelamente, continuaron con la ofensiva en las salas de justicia, favorecida desde 1882 por la nueva ley de enjuiciamiento criminal, que contemplaba la celebración de juicios orales y públicos y permitía la presencia de curiosos y de la prensa, de manera que esta última acabó jugando un papel de primer orden en la difusión de las nuevas ideas que relacionaban la locura y la criminalidad (Seoane, 1989; Trinidad Fernández, 1991; Campos, 2012, 35-40, 131-136).

Los procesos de Manuel Morillo (1884), acusado de asesinar a la madre de su novia y de herir gravemente al padre (Campos, 2012), el del cura Galeote, asesino del Obispo de Madrid (1886) (Varela, Álvarez-Uría, 1979; Campos, 2003), el de Hillairaud (1887), que intentó asesinar al Mariscal Bazaine en Madrid (Sánchez de Ocaña, 1887; Escuder, 1895, 107-129), o el del escritor Remigio Vega Armentero (1889), que mató a su esposa por celos (Fernández, 2001), se sucedieron durante toda la década y dieron la oportunidad, entre otros, a Jaime Vera, Luis Simarro y José María Escuder, de participar como peritos y defender la vinculación entre locura, criminalidad, herencia patológica y anormal conformación orgánica. Escuder destacará sobre todos ellos en la defensa de estos postulados. Sus informes periciales en los casos de Morillo, Galeote e Hillairaud son un ejemplo de utilización escolástica y dogmática de los postulados degeneracionistas y las teorías lombrosianas (Álvarez-Uría, 1983; Campos, 2012). Sus peritajes se caracterizaban por la construcción de árboles genealógicos llenos de enfermos mentales y anormales que transmitían sus padecimientos generación tras generación hasta desembocar en el loco criminal que se juzgaba. Asimismo, las descripciones físicas de los procesados que realizó eran profundamente expresivas y tenían tintes novelescos. Cuerpos maltrechos, rostros deformes, cráneos anormales, aspectos simiescos, frentes de reptil o estrabismo, son, de hecho, algunos de los estigmas recurrentes en sus peritajes.

Como trasfondo de estos cambios, hay que considerar que, tradicionalmente, la locura no había sido considerada como una enfermedad en sentido estric- to, sino más bien como un desorden del espíritu (Renneville, 2003). La tarea que acometieron los primeros alienistas fue medicalizarla, transformándola en una enfermedad. Para ello, combatieron el concepto popular de locura, que comportaba una pérdida completa de la razón y se manifestaba por un delirio fácilmente perceptible a los ojos de los profanos. Esta era la concepción que a lo largo del siglo XIX defendía la mayoría de la judicatura española, que insistía en que para "deslindar la normalidad de la locura» no era necesario el "criterio psiquiátrico» sino «la evidencia percibida por la razón, los sentidos» y, en definitiva, por «el sentido común» (González González, 1994, 39). A esta concepción de la locura los frenópatas contrapusieron un sistema de clasificaciones nosográficas que ampliaba notablemente el concepto de locura y cuya comprensión requería el buen ojo clínico del nuevo experto en la materia.

Esta tensión, presente en todos los foros de discusión, se hizo patente en los juicios y, de manera especial, en los turnos de preguntas y respuestas que seguían a las exposiciones periciales, percibiéndose nítidamente los intentos de los juristas de desmontar los argumentos científicos de los alienistas al subrayar sus contradicciones y la inconsistencia de muchos de sus argumentos. Esta oposición doctrinal en torno a la enfermedad mental dio lugar a la construcción de dos retratos del sujeto peligroso. La psiquiátrica ahondaba en el componente patológico y en la inquietante idea de la existencia de "locos que no lo parecen» que entrañaban un peligro latente. La jurídica negaba los elementos patológicos y la existencia de una estructura morbosa de la personalidad, remitiendo a comportamientos y situaciones familiares desviadas de las normas que se traducían en concepciones erróneas de la realidad social.

Por otra parte, los peritos se encontraban con trabas jurídicas para exponer sus criterios científicos ante los tribunales. En algunos juicios como el de Morillo, el presidente del tribunal les prohibió referirse a acontecimientos que no se hallasen comprobados en el sumario, debiendo circunscribir sus peritajes al estado mental del acusado en el momento de cometer su crimen. Esta delimitación les impedía explicar el origen de la enfermedad mental y referirse a la herencia patológica (Campos, 2012, 158-160). Sin embargo, la práctica muestra que la exposición de los cuadros patológicos de las familias por parte de los peritos acabó siendo tolerada en los tribunales de justicia.

No obstante, y pese a las prohibiciones de los jueces, tanto la defensa como la fiscalía intentaban averiguar si los acusados habían mostrado síntomas de locura con anterioridad a su crimen (Foucault, 2001, 28). Así, en los juicios de Morillo o Galeote, las preguntas de la fiscalía se dirigieron a averiguar si podían 
existir intervalos de razón en sus supuestas locuras, mientras que la defensa intentaba que los testigos corroborasen que la alienación mental venía de antiguo. En estos interrogatorios se percibe el peso de la visión tradicional de la locura, pues tanto las preguntas como las respuestas partían de la premisa de que cualquiera podía percibir la locura de un individuo. Pero, en algunos casos, se iba más lejos, y los testigos llegaban a establecer la conexión hereditaria de la patología traspasando los límites marcados por la ley. Así, por ejemplo, en el juicio del cura Galeote, el abogado defensor preguntó a José García, que conocía de antiguo al acusado, si sabía de "algún individuo de la familia del procesado que esté loco», respondiendo este que:

sí, que Gabriel está medio loco, y Daniel murió en concepto de demente, y algún individuo de la familia del procesado por parte de madre, encuéntrase en estado de idiotismo y le llaman vulgarmente el tonto cotilla. Dice respecto a Galeote, que tan arraigada es su creencia de que se halla loco, que la última vez que lo vio no pudo menos de exclamar al separarse: -Pobre Cayetano! ¡Otro Daniel! (Varela, Álvarez-Uría, 1979, 98)

En el polo opuesto estaría el argumento del fiscal del proceso del célebre crimen de la calle de San Hermenegildo, que, ante la posibilidad de que el reo fuera epiléptico, concluía que la apelación a la locura de los acusados se había convertido en una moda y defendía que "hay hechos aislados, grandes crímenes cometidos sin ningún motivo aparente, sin que se conozcan las causas que de ordinario explican, sin justificarla, la acción criminal» (Sáez Domingo, 1885, 111). Pero esos motivos desconocidos no tenían que responder necesariamente a una patología mental, sino a una personalidad malvada y cruel. En una línea similar, aunque en un tono más conciliador y matizado, el fiscal del proceso Hillairaud consideraba que el "procesado", por su educación, hábitos y "falta de sólidas creencias», había dedicado su «vida a la satisfacción de sus instintos materiales», dejándose llevar por sus pasiones "sin freno" y sus impulsos desordenados, hasta el punto de cometer su crimen (Sánchez de Ocaña, 1887, 43).

Llegados a este punto, conviene reflexionar sobre el calado de los peritajes psiquiátricos. Michel Foucault ha señalado con acierto que los peritajes traspasaban la línea marcada por la ley penal, que solo permitía juzgar delitos previamente establecidos y recogidos en el Código Penal. Los peritajes, en este sentido, introducían elementos laxos que se referían a los comportamientos de los encausados, integrando su acto en la conducta global del individuo. "La pericia permitiría constituir un doblete psicológico ético del delito", poniendo de relieve no una «infracción en el sentido legal del término, sino una irregularidad con respec- to a una serie de reglas que pueden ser fisiológicas, psicológicas o morales». Además, los peritajes intentaban demostrar "cómo el individuo se parecía ya a su crimen antes de haberlo cometido», construyendo a partir de lo que denomina «ilegalidades infraliminares» una «reconstrucción anticipatoria del crimen» (Foucault, 2001, 27-28). En este sentido, los peritajes, con su lenguaje pueril y sus ambigüedades, construirían un individuo moralmente defectuoso y con comportamientos peligrosos (Foucault, 2001, 28-30).

De las dos concepciones sobre el individuo criminal enfrentadas se extraían consecuencias jurídicas que prolongaban la discusión en otros ámbitos. Frente a los principios defendidos por los juristas sobre la responsabilidad penal del individuo, que solo contemplaba la locura como eximente en determinados casos, los alienistas esgrimieron los argumentos degeneracionistas y lombrosianos para defender la irresponsabilidad penal de los locos criminales, negando la existencia del libre albedrío. Así, Victoriano Garrido, próximo a Esquerdo, consideraba que el libre albedrío era una "rémora del progreso" frente a la que se alzaban las «límpidas aguas de la escuela positivista» (Garrido, 1888, 6-9), y afirmaba que los estudios de Ferri y Lombroso absorbían en ese momento de tal modo la atención de los jurisconsultos, médicos y legisladores que resultaba imposible sustraerse a «esa corriente general que impregna a la manera de suave rocío el espíritu contemporáneo» (Garrido, 1888, 230).

Existe un cierto consenso historiográfico en considerar 1887 como el año de la consolidación de la Antropología criminal en España (Maristany, 1973; Galera, 1991; González González, 1994, 181-182). Los peritajes psiquiátricos habían contribuido notablemente a abonar el terreno para su eclosión, familiarizando a los tribunales y a la opinión pública con la terminología del degeneracionismo y con la idea de la existencia de individuos azotados por la locura cuyo aspecto físico y herencia patológica les delataban como peligrosos. Desde comienzos de la década, la Antropología criminal había comenzado a ser conocida en España, sucediéndose los trabajos que la defendían, así como los peritajes que se basaban en sus principios (González González, 1994, 180). Pero fue en 1887 cuando la notoriedad de los peritajes psiquiátricos en la difusión del degeneracionismo y la Antropología criminal provocó airadas respuestas de los magistrados a estas nuevas ideas sobre el crimen. Ese año, el Presidente del Tribunal Supremo finalizó su discurso de apertura de los tribunales enfatizando que estos rechazarían taxativamente las nuevas doctrinas positivas por su carácter destructor de todo sistema social (Maristany, 1973). El enfrentamiento entre juristas y médicos sobre la responsabilidad penal del loco delincuente y sobre la naturaleza del criminal alcanzó uno de sus puntos culminantes en el largo y apasionado debate 
sobre «Los médicos alienistas y los tribunales de justicia» que tuvo lugar en la Academia Médico Quirúrgica durante el primer semestre de 1887, y que alcanzó su máxima intensidad con la amarga disputa entre José María Escuder y el Secretario de la Real Academia de Jurisprudencia, Luis Arquiola (Campos, 2012, 237-246).

Sin embargo, hubo críticas más inteligentes, como las del catedrático de derecho penal de la Universidad de Oviedo Félix Aramburu. Seguidor de las tesis correccionalistas, en 1887 publicó La nueva ciencia penal (exposición y crítica), donde refutaba las ideas lombrosianas. Pese a su abierta crítica, fue la primera exposición pública y minuciosa de la Antropología criminal en España. Aramburu defendía el derecho penal clásico y descalificaba la Antropología criminal, contraponiendo al determinismo biológico y a las innovaciones penales de la misma la existencia del libre albedrío y la proporcionalidad de las penas (Maristany, 1973; Campos, Huertas, 2013).

No obstante, en la década de 1880 el debate no se centró tanto en la peligrosidad del loco criminal como en demostrar su irresponsabilidad penal y en cuestionar la existencia del libre albedrío. La utilización de conceptos propios de la Antropología criminal, entremezclados con el degeneracionismo, no pretendía mostrar la existencia de un criminal nato, sino la vinculación entre locura, herencia biológica, defectuosa conformación corporal e irresponsabilidad penal. De hecho, era un discurso con una fuerte carga filantrópica, contrario a la pena de muerte y favorable, al menos en su retórica, al tratamiento humanitario del loco criminal, por lo que se alejaba de los principios de defensa social de la escuela italiana.

El trasfondo de las discusiones entre psiquiatras y juristas en los juicios fue la tensión entre una visión esencialista de la locura, defendida por los peritos, y otra, defendida por los juristas, que podríamos calificar de locura codificada, que no intentaba definir la locura, sino cotejar cada caso particular con el Código Penal. La primera visión preconizaba que cualquier individuo que padeciera una patología mental, por ligera que fuera, y cometiera un delito debía ser considerado irresponsable a efectos penales. Esta posición la defendió con contundencia Esquerdo en 1882 ante la Comisión del Senado encargada de estudiar la reforma del Código Penal (López, 1882). La posición de los juristas estaba bien ejemplificada en las conclusiones del fiscal del proceso Hillairaud cuando señalaba que, aunque pudieran existir "estadios intermedios, locuras parciales» y, en general, formas de locura que no respondieran a estados absolutos «de falta de razón, de delirio" y de "demencia declarada», no podían ser tomados en consideración, porque el Código Penal no los recogía como tales (Sánchez de Ocaña, 1887, 5455). No obstante, el debate todavía no se ceñía a la peligrosidad predelictual del loco criminal, a pesar de que esta estaba latente. En 1885 Raffaele Garofalo se refirió a la temibilidad y peligrosidad de los delincuentes como los conceptos sobre los que debía construirse una nueva penalidad que rompiese con la escuela penal clásica (Garofalo, 1885). A comienzos del siglo $\mathrm{XX}$, esta idea se abriría paso de forma generalizada.

\section{DEL DEGENERADO AL PELIGROSO SOCIAL}

En el juicio de Hillairaud, el fiscal planteó la imposibilidad de que el marco penal español pudiera tomar en cuenta los estadios intermedios de la locura. Sin embargo, a mediados de la década de 1890 algunos psiquiatras intentaron aproximar posiciones con los juristas defendiendo la existencia de la «responsabilidad atenuada» en muchos degenerados.

En 1894, Vicente Ots y Esquerdo publicó un folleto titulado La locura ante los tribunales en el que atribuía las discrepancias entre "magistrados y frenópatas de nuestro país» a los excesos de la doctrina "ultra-radical» - representada por el grupo de Esquerdo- que defendía un concepto demasiado extenso de los trastornos psicopáticos y de la eximente de responsabilidad penal (Ots y Esquerdo, 1894, 19). Ots consideraba que la noción lombrosiana del criminal nato perjudicaba la aceptación del degeneracionismo en los tribunales. En su opinión, "la especie humana, al sufrir degeneración», recorría "dos trayectorias divergentes»: la degeneración vesánica, que terminaba en el manicomio, y la degeneración criminal, que llevaba al individuo al presidio (Ots y Esquerdo, 1894, 11). Las consecuencias de ambas degeneraciones en relación con la responsabilidad penal debían ser distintas. El loco era un enfermo que obraba "por impulsos morbosos, por determinaciones patológicas», y producía el mal "por el fatalismo de su organización, que forzosamente le impulsa a reaccionar de un modo insano", por lo que debía ser considerado irresponsable. Sin embargo, el criminal era "un ser depravado, parético, un microbio», cuyas malas acciones eran originadas por «el desenvolvimiento que han tenido sus morbosas tendencias instintivas, en una atmósfera pestilente». En este sentido, era responsable de todas sus acciones delictivas, por ser estas «el resultado de su educación perniciosa» (Ots y Esquerdo, 1894, 11-12). Su propuesta estaba dirigida a buscar un pacto entre magistrados y psiquiatras para acabar con el conflicto en los tribunales. Para lograrlo, proponía que se aceptara la doctrina de la responsabilidad atenuada, que defendía la responsabilidad de los enfermos mentales cuando sus actos criminales se cometían fuera de la esfera de su patología mental. En este sentido, los degenerados caerían en muchos casos dentro de la justicia penal al ser responsables de sus actos delictivos, siempre y cuando estos no se produjeran en "la esfera de sus impulsos morbosos» (Ots y Esquerdo, 1894, 50). 
En una línea similar se situaba Luis Dolsa, que consideraba que desde Pinel se había producido un ensanchamiento de las fronteras de la irresponsabilidad que alarmaba a la sociedad y pervertía la aplicación del Código Penal. Frente a este estado de cosas, argumentaba que ya no bastaba que:

el individuo esté afecto de alienación mental en el momento de la acción punible; es necesario [...] que la alienación sea bastante grave y permanente para quitar al individuo afecto toda libertad moral y con ella toda posibilidad de resistir el impulso de su estado morboso. De ahí que la responsabilidad parcial sea un hecho en psiquiatría que, como sabéis, consiste en que un mismo individuo puede cometer actos responsables o irresponsables, según que entren en

la esfera de la razón o del delirio (Dolsa, 1895, 42).

Los degenerados entraban de lleno en esta categoría, pues, debido a la extensa gradación de la degeneración, había "fases en donde cabe el castigo, fases donde se atenúa y fases en las cuales no debe aplicarse» (Dolsa, 1895, 40). Asimismo, frente a Lombroso y su escuela, negaba que la degeneración fuera un elemento "etiológico fatalmente seguro de la criminalidad», o que el crimen fuera un «signo patognomónico de dolencia alguna» (Dolsa, 1895, 55). No obstante, pese a estas críticas, el degeneracionismo y los postulados de la Antropología criminal continuaron defendiéndose en los peritajes psiquiátricos.

En cualquier caso, a comienzos del siglo XX se produjeron algunos cambios en las posiciones de los psiquiatras que originaron una redefinición en las relaciones entre crimen y la locura y en la manera de abordarlas penalmente. El debate comenzó a articularse en torno a la idea de la potencial peligrosidad social de los locos y degenerados y en los medios para detectarla antes de la comisión de un delito. Se abandonaba, por tanto, la retórica filantrópica y las posiciones contrarias a la pena de muerte que habían caracterizado a los primeros psiquiatras degeneracionistas en favor de una concepción que no descartaba la eliminación del sujeto peligroso como medida de defensa social.

La trayectoria del médico legista Lecha-Marzo da la medida del cambio operado a principios del siglo XX (Martínez-Pérez, 1986; Martínez-Pérez, 1989). Degeneracionista convencido, en 1906 publicó un trabajo titulado "Las anomalías de Mateo Morral» en el que trataba de suministrar algunos datos sobre las alteraciones reveladas por la autopsia del cadáver de este anarquista que había atentado contra el cortejo nupcial de Alfonso XIII y Victoria Eugenia. Aunque LechaMarzo sostenía que «el progeneismo, la prominencia de los senos frontales y la desviación de la nariz [...] hacen de Morral un tipo criminal de Lombroso» (Lecha-Marzo, 1906, 87), también señalaba que no era irresponsable porque en su cerebro no se había encontrado "ninguna anomalía de carácter atávico» y estaba «macroscópicamente bien conformado» (Lecha-Marzo, 1906, 88). Ante la posibilidad de que fuera declarado irresponsable si se patologizaba su conducta, Lecha-Marzo le consideró como un mero delincuente al que se le debía aplicar la pena que le correspondiera sin ningún eximente. Rompía así con una tradición fuertemente arraigada en la psiquiatría y la criminología consistente en descalificar los movimientos sociales y políticos por medio de su patologización, estableciendo una relación directa entre la subversión, la locura y el crimen (Álvarez-Uría, 1983, 244260; Campos, 1995; Girón, 2002). La patologización de la protesta social perseguía minimizar su importancia, atribuyendo toda su responsabilidad a líderes o grupos de anormales y desviados, descalificando los comportamientos e ideas políticas que ponían en entredicho el orden social. De esta manera, se evitaba afrontar los problemas sociales desde una posición política y buscar las soluciones adecuadas.

Consciente de que la aplicación ortodoxa del degeneracionismo podía suponer un freno para ejercer la defensa social, en 1911 Lecha-Marzo propuso un nuevo escenario en el que estas doctrinas debían ser ajustadas (Lecha-Marzo, 1911a). A su juicio, mientras el principio de libre albedrío y la cuestión de la responsabilidad o irresponsabilidad del delincuente fueran un elemento fundamental sobre el que basar las penas, un gran número de delincuentes podrían, gracias a informes psiquiátrico-forenses que demostraran la existencia de un trastorno mental, beneficiarse de la responsabilidad atenuada. De ahí se derivaría que el encierro en prisión se vería recortado en cuanto a su duración. La reacción frente a esta posibilidad fue contundente. Junto a la buena aplicación de los saberes antropológico criminales, Lecha-Marzo propugnó una medida tajante y polémica para evitar esos efectos negativos, a saber, "no disminuir la pena en los irresponsables parciales» (Lecha-Marzo, 1911b, 101), añadiendo que «al tratarse de sujetos menos aptos que los normales para pesar el pro y el contra de los actos que realizan», y por poseer "una voluntad completamente inestable», se hacía preciso reforzar esa voluntad mediante una elevación de la pena «en razón directa» con el grado de inestabilidad al objeto de "producir en su cerebro un efecto eficaz» (LechaMarzo, 1911b, 102).

Esta línea de pensamiento debe encuadrarse en los esfuerzos de médicos y juristas lombrosianos por lograr una reforma profunda de los códigos penales que sustituyera el concepto de "responsabilidad» por el de «peligrosidad social». En un trabajo de 1915 dedicado a la "prueba médica del discernimiento», LechaMarzo y Antonio Piga expresaban con claridad esta nueva tendencia: 
Lo que debían pretender los tribunales [...] es averiguar si el sujeto acusado es temible o no para la sociedad en que vive. El concepto de temibilidad, como sustituto del de libre albedrío, se abre paso rápidamente (Lecha-Marzo, Piga, 1915, 307-308).

Por tanto, para determinados sectores de la ciencia, conocer el grado de libertad moral con el que actuaba el individuo delincuente ya no era la cuestión fundamental a debatir en las salas de justicia. Lo importante para la seguridad de la sociedad era establecer si se le podía considerar "peligroso». Solo así podría ejercerse una adecuada defensa social que pasaba por caracterizar al delincuente tanto somática como psíquicamente, y por descubrir con los mismos procedimientos a aquellos sujetos sospechosos de poder llegar alguna vez a atentar contra el orden establecido.

\section{DEL INDIVIDUO PELIGROSO A LA SOSPECHA GENERAL: LA HIGIENE MENTAL}

Paralelamente a estas transformaciones en el marco de la psiquiatría legal, la criminología de comienzos del siglo XX comenzó a fijar su atención en el análisis de la pequeña delincuencia y de la vida maleante más artesanal de las grandes ciudades, cuya existencia resultaba inquietante socialmente por su carácter y dificultad de aprehensión, dando lugar a los estudios sobre la "mala vida» (Maristany, 1998, XXXV). De este modo, el interés de la criminología también se centró en los individuos con conductas delictuales que formaban parte de los territorios más cotidianos de la imagen de la anormalidad y del desequilibrio (Cleminson, 2009; Campos, 2009). En España, los trabajos más importantes en este sentido fueron La mala vida en Madrid de Constancio Bernaldo de Quirós y José María Llanas de Aguilaniedo, publicado en 1901, y La mala vida en Barcelona, de Max-Bembo. Según Foucault, las tesis degeneracionistas hicieron innecesaria la oposición entre el crimen monstruoso y la pequeña criminalidad, estableciendo un continuum en el que todo el territorio de las infracciones quedaba bajo la mirada científica permitiendo relacionar «al menor de los criminales con un peligro patológico para la sociedad, para la especie humana en su conjunto» (Foucault, 1990b, 251-252). El discurso sobre la " mala vida", inserto en el cruce de caminos entre el degeneracionismo y la criminología positivista, pretendía analizar un estrato de la población de las grandes urbes marcado por la desviación de las conductas, la anormalidad psíquica y social y la proximidad al delito (Cleminson, Fuentes Peris, 2009). Una variopinta gama de individuos y grupos marginales como prostitutas, homosexuales, mendigos, vagabundos, estafadores, golfos, gitanos, etc., fueron el objeto de estudio de estas obras. Se trataba de analizar y catalogar a una población, considerada peligrosa y patológica por sus comportamientos desviados, para reconducirla, normalizarla y gobernarla. Este tipo de planteamientos llevó a naturalizar el crimen y los comportamientos desviados a partir de criterios claramente económicos y sociales. El ejemplo más evidente de ello fue la utilización de la falta de trabajo y la miseria como elementos decisivos para establecer la frontera entre la normalidad y la anormalidad. La falta de trabajo fue así patologizada y criminalizada, convirtiendo a una parte importante de la población en sospechosa de comportamientos antisociales y peligrosos. La importancia de este tipo de estudios residía en que ensanchaba el campo de la peligrosidad social y ahondaba en la idea de la profilaxis del delito y de la necesidad de imponer sentencias indeterminadas. En este sentido, los trabajos sobre la mala vida tuvieron años después, una notable influencia en la elaboración de la Ley de Vagos y Maleantes.

En las primeras décadas del siglo $\mathrm{XX}$, en el terreno jurídico, tradicionalmente refractario a los cambios provenientes de la psiquiatría y de la criminología, también comenzaron a producirse transformaciones tendentes a considerar la defensa social y la peligrosidad predelictual como bases del derecho penal. El fenómeno no fue particular de España, sino que formaba parte de la expansión del movimiento de «defensa social» en Europa y América surgido del debate criminológico de finales del siglo XIX. La creación en 1889 de la Unión Internacional de Derecho Penal fue un hecho fundamental en la extensión del debate sobre la peligrosidad y sus consecuencias penales. Frente a la idea del castigo equitativo y retributivo defendido por la escuela penal clásica, se fue imponiendo, impulsada por la Antropología criminal, la idea de la aplicación de castigos personalizados en función de la naturaleza del criminal. Pero la noción de peligrosidad predelictual se acompañó del desarrollo de otro concepto de largo recorrido, el de las sentencias indeterminadas. Hacia 1910 ambos asuntos (peligrosidad y sentencias indeterminadas) eran el centro del debate jurídico y criminológico (Kalifa, 2005, 260-265); ese año, el jurista Adolphe Prins publicó La Défense sociale et les transformations du droit pénal, en el que afirmaba la existencia del estado de peligrosidad sin delito y el derecho del estado a intervenir en esos casos de manera preventiva (Prins, 1910,76).

En España, las discusiones sobre la peligrosidad social de los individuos también marcaron los debates criminológicos y psiquiátricos del primer tercio del siglo $\mathrm{XX}, \mathrm{y}$ adquirieron una especial relevancia en las décadas de 1920 y 1930 de la mano del movimiento de higiene mental. Además, la conceptualización psiquiátrica de la peligrosidad confluyó, especialmente durante la II República, con una nueva conceptualización jurídica y penitenciaria de la misma y con una enorme preocupación de los gobiernos republicanos por el mantenimiento del orden público, lo que se tradujo en la promulgación de leyes fuertemente repre- 
sivas como la Ley de Defensa de la República y la Ley de Orden Público (Ballbé, 1985, 317-396; Casanova, 1997, 18-22). Asimismo, también se aprobaron leyes, como la Ley de Vagos y Maleantes de 1933, encaminadas a la prevención de la delincuencia basadas en la peligrosidad potencial de determinados individuos. Por tanto, las reformas psiquiátrica y penitenciaria emprendidas durante el primer bienio republicano se entrecruzaron creando espacios comunes en los que se hacía difícil distinguir lo penal y lo psiquiátrico. Ejemplos de esta intersección fueron el Instituto de Estudios Penales, fundado en marzo de 1932, y el Servicio de Biología Criminal, creado en febrero de 1933, en los que compartían responsabilidades psiquiatras y juristas. El nombramiento en 1933 del psiquiatra Manuel Ruiz Maya como Director General de Prisiones personifica esta convergencia de intereses. Su breve mandato coincidió con la creciente conflictividad social y política de la sociedad española y con la elaboración y aprobación de La Ley de Vagos y Maleantes y la Ley de Orden Público (Gargallo, 2011). Precisamente, Ruiz Maya fue uno de los más destacados teóricos de la peligrosidad predelictual desde el punto de vista psiquiátrico, como lo muestran sus intervenciones en las reuniones anuales de la Asociación Española de Neuropsiquiatría (AEN) de 1927 y 1928, y la publicación de su monumental Manual de psiquiatría penal y civil en 1931 (Campos, 2007).

En 1927, Ruiz Maya presentó en la Segunda Reunión Anual de la AEN un trabajo titulado "La peligrosidad de los alienados en sus aspectos teórico y práctico» en el que afirmaba que, desde el punto de vista psiquiátrico, la peligrosidad se confundía con «el concepto de enfermedad mental». A su juicio, la peligrosidad residía más en la potencialidad de cometer actos peligrosos que en su comisión. Además, señalaba que la peligrosidad no podía limitarse a la contravención potencial o efectiva de las normas jurídicas, sino que debía extenderse a "cuanto pueda vulnerar la totalidad de las normas habituales de vida» (Ruiz Maya, $1928,6)$. De este modo, la peligrosidad no se reducía a la comisión de los delitos estipulados por la ley, sino que se extendía a la vulneración de la moral pública «no sujeta sino a la reglamentación tácita del hábito o de la costumbre, y a cuanto la constituye o informa» (Ruiz Maya, 1930, 59). Estas infracciones o reacciones, tildadas como "paralegales», eran más importantes que las delictivas y precisaban de mecanismos de prevención del delito. En este sentido, Emilio Mira señalaba que la "verdadera finalidad» del Derecho debería ser "evitar la delincuencia», insistiendo en que, para lograrlo, la acción jurídica tenía que desbordar «el estrecho campo de la acción penal para lanzarse en el fértil terreno de la higiene social y más concretamente de la profilaxis delictiva» (Mira y López, 1932, 236).

El concepto de peligrosidad predelictual y la prevención situaba a la psiquiatría ante el desafío de implementar algún tipo de tecnología que permitiera averiguar científicamente quienes eran los individuos peligrosos. Aunque hubo intentos de establecer los parámetros de la peligrosidad potencial de manera científica (Campos, 2007, 23-24), los psiquiatras optaron no solo por «estudiar la peligrosidad de los enfermos mentales, sino también la de los individuos normales, con reacciones caracterológicas violentas, porque al fin este es un problema psicológico-psiquiátrico para juzgar del cual ningún otro profesional está tan capacitado» (Rodríguez Lafora, 1929, 67). Por tanto, a comienzos de los años treinta, la psiquiatría había pasado de definir al enfermo mental como peligroso a proponer el estudio de todas las personas sanas en busca de su potencial peligrosidad. En un contexto caracterizado por la conversión de la higiene y la profilaxis mental en el eje de actuación de la psiquiatría, que extendía y justificaba su acción en el seno de la sociedad proponiendo investigar y controlar científicamente a los ciudadanos, la exploración de la potencial peligrosidad de los individuos constituía el epicentro del programa de higiene mental que defendían los psiquiatras. En estas coordenadas, la normalidad, en palabras de Castel, era sospechosa «de no ser más que una apariencia», teniendo que "aportar pruebas ante un tribunal de especialistas en patología» (Castel, 1980, 193-194) $)^{3}$.

Sin embargo, conscientes de las dificultades de estudiar a toda la población la mayoría de los psiquiatras defendían la implantación del reconocimiento psicofísico de todos los delincuentes como medida de defensa social que permitiera diferenciar a los criminales enfermos de los normales y poder actuar en consecuencia. Además argüían que el examen profundo de los delincuentes no alienados era fundamental para «inquirir su grado de peligrosidad y corregibilidad» y poder conocer «el tipo biológico de su personalidad y los factores etiológicos que han motivado su reacción antisocial» (Saforcada, Torras, 1930, 453). En esta línea, en 1933 se creó en Madrid el Servicio de Biología Criminal -encargado de «el estudio científico sistemático de todos los delincuentes que se hallen recluidos en las prisiones de Madrid»-con el argumento de que el estudio científico de la personalidad del delincuente podía aportar datos

sumamente valiosos, de evidente trascendencia práctica, que no solo permitan un pronóstico social, sino que, al mismo tiempo proporcionen el material básico para organizar de un modo severamente científico la profilaxis de la criminalidad ${ }^{4}$.

Asimismo, también en el terreno de la codificación penal se produjeron cambios. La dictadura de Primo de Rivera promulgó en 1928 un nuevo Código Penal, que pese a recoger algunos postulados de la psiquiatría y la criminología sobre la peligrosidad social, fue criticado 
porque no satisfizo los planteamientos de los psiquiatras sobre la prevención del delito ni sobre las medidas de seguridad que debían aplicarse a los sujetos potencialmente peligrosos. El Código de 1928 fue anulado por el Gobierno de la República el 15 de abril de 1931. Hasta que se promulgara un nuevo Código, se restituyó el de 1870, adaptándolo a la legalidad republicana. En el terreno psiquiátrico, gracias a la intervención de José Sanchís Banús, se modificó el artículo 8o, permitiendo una interpretación más laxa de la locura que calmaba a los psiquiatras en tanto llegaba el futuro Código:

Están exentos de responsabilidad criminal: 1으 El enajenado y el que se halle en situación de trastorno mental transitorio, a no ser que este haya sido buscado de propósito, para que la embriaguez exima de responsabilidad ha de ser plena y fortuita.

La reforma daba una mayor flexibilidad a las interpretaciones de los casos de locura, pero no introducía ninguna de las reivindicaciones esgrimidas por los psiquiatras sobre la prevención del delito o las medidas de seguridad a adoptar frente a delincuentes e individuos potencialmente peligrosos.

Estas vendrían de la mano de la célebre Ley de Vagos y Maleantes del 4 de agosto de $1933^{5}$. Esta ley tuvo un complejo proceso de elaboración, pues los socialistas se opusieron a su primer borrador. El gobierno, deseoso de sacarla adelante, accedió a su revisión y encargó su redacción a los diputados y juristas Luis Jiménez de Asúa del PSOE y Mariano Ruiz Funes de Acción Republicana, profundos conocedores y partidarios ambos de las nuevas corrientes jurídicas y criminológicas (Campos, 2007). Como gran novedad, la ley introducía la peligrosidad predelictual, que, según Jiménez de Asúa, significaba la «vehemente presunción de que una determinada persona quebrantará la ley penal» (Jiménez de Asúa, 1934, 33). Asimismo, definía varias categorías de peligrosidad predelictual y proponía las medidas que debían tomarse contra este tipo de individuos. La mayor parte de los individuos catalogados como vagos y maleantes coincidía plenamente con los estudiados por la literatura criminológica de la «mala vida». Así, incluía a «los vagos habituales», "los rufianes y los proxenetas», "los mendigos profesionales», "los ebrios y toxicómanos» y los que vivieran de «la mendicidad ajena o explotasen menores de edad o enfermos mentales». También contemplaba otras categorías de peligrosidad sin delito como eran la imposibilidad de justificar el domicilio, la ocultación de la verdadera identidad, la posesión de documentos de identidad falsos, el quebrantamiento de una orden de expulsión del territorio nacional por los extranjeros, la no justificación de la posesión de dinero o bienes a requerimiento de las autoridades, así como la explotación de juegos prohibidos y la provisión de bebidas alcohólicas a menores de 14 años. Además, se consideraba como caso de peligrosidad predelictual «mantener trato asiduo o frecuentar lugares en los que se dieran cita maleantes». Por tanto, la Ley de Vagos y Maleantes etiquetaba como peligrosos a los individuos que no vivían de su trabajo, estableciendo, como era habitual desde hacia décadas, la relación con este como principal indicador de normalidad social. Las medidas de seguridad que debían aplicarse a estos «sujetos peligrosos» consistían principalmente en el encierro del individuo en establecimientos correctivos o curativos acordes con su estado de peligrosidad; en fijar su pertenencia a un territorio, obligándole a demostrar un domicilio fijo; y, por último, en someterse a la vigilancia e indicaciones de los delegados asignados por la autoridad. La ley dejaba al criterio del juez la imposición de la duración de la medida de seguridad dentro de un límite establecido, consagrando la flexibilización de la pena.

Desde su proceso de elaboración la ley suscitó temores por la posibilidad de que fuera aplicada indiscriminadamente. Tanto Ruiz-Funes como Jiménez de Asúa negaron este extremo, afirmando que no existía peligro de una aplicación desmedida de la misma. (Heredia Urzaiz, 2006; Heredia Urzaiz, 2009; Gargallo, 2011). Igualmente, Jiménez de Asúa insistió en que la ley no atentaba contra el liberalismo sino que lo reforzaba, porque terminaba con prácticas policiales al margen del derecho para perseguir a los malvivientes (Jiménez de Asúa, 1934, 64). Sin embargo, la Ley de Vagos y Maleantes fue utilizada muy pronto como un instrumento de represión generalizada a militantes de partidos políticos molestos, endureciéndose su aplicación tras los sucesos revolucionarios de octubre de 1934 (Heredia Urzaiz, 2006; Gargallo, 2011). Además, en noviembre de 1935, a instancias de la mayoría derechista de la CEDA, se incluyó un nuevo supuesto de peligrosidad sin delito: el de propaganda y actividades sociales que incitasen reiteradamente «a la ejecución de delitos de terrorismo, de atraco y los que públicamente glorifiquen o enaltezcan la comisión de dichos delitos ${ }^{6}$. En este sentido, algunos diarios informaron del uso abusivo de la ley contra militantes de izquierdas, llegándose a denunciar que se hubiera llegado a aplicar a obreros que poseían libros de contenido social avanzado ${ }^{7}$. El propio Jiménez de Asúa se mostró escandalizado por esta manera de aplicar la ley e intentó salvar su prestigio recordando que era una ley «científica dirigida a combatir a la peligrosidad potencial de los vagos y maleantes $»^{8}$.

Pese a todo, la ley se mantuvo en vigor durante el franquismo hasta que fue sustituida por la Ley de Orden Público de 1970. En 1954 se introdujo a los homosexuales como sujetos susceptibles de ser declarados peligrosos, y se añadió un párrafo que hacía extensiva su aplicación a «los que de cualquier manera, perturben con su conducta o pusieren en peligro la paz social o la tranquilidad pública». Y, finalmente, en 1958 
se crearon dos plazas de médicos forenses adscritas a los nuevos juzgados especiales de vagos y maleantes, argumentando que la declaración de peligrosidad de los sujetos comprendidos en el artículo segundo «requiere en muchos casos la intervención de esos

\section{NOTAS}

1 Trabajo realizado en el marco del proyecto de investigación HAR2009-13389-C03-02.

2 Las dos primeras, dictadas el 12 de marzo y el 10 de abril de 1880 se referían a Manuel Otero, mientras que las siguientes, leídas el 25 de enero y el 2 de febrero de 1881 estaban dedicadas al "Sacamantecas".

3 Castel, señalaba que el concepto de monomanía acuñado a comienzos del siglo XIX inauguró "una era de sospecha generalizada." Desde nuestro punto de vista, en el caso español su reflexión es aplicable sólo a partir de las décadas de 1920 y 1930.

4 Decreto creando en el Instituto de Estudios Penales, con carácter de ensayo, un Anexo psiquiátrico, en el que figurará un Servicio de Biología Criminal, adscrito y dependiente de dicho Instituto. Gaceta de Madrid núm. 73, de 14 de marzo de 1933, p. 1964.

5 "Ley de vagos y maleantes". Gaceta de Madrid, 5 de agosto de 1933, núm 217.

\section{BIBLIOGRAFÍA}

Álvarez-Uría, Fernando (1983), Miserables y locos. Medicina Mental y orden social en la España del siglo XIX, Madrid, Tusquets Editores.

Aramburu Zuloaga, Félix (1887), La nueva ciencia penal, Madrid, Est. tip. de Ricardo Fe.

Ballbé, Manuel (1985), Orden público y militarismo en la España constitucional (1812-1983), Madrid, Alianza Editorial.

Bernaldo de Quirós, Constancio y Llanas Aguilaniedo, José María (1901), La mala vida en Madrid. Estudio psico-sociológico con dibujos y fotograbados del natural, Madrid, B. Rodríguez Serra Editor.

Campos Marín, Ricardo (1999), "La teoría de la degeneración y la profesionalización de la psiquiatría en España (1876-1920)”, Asclepio, LI (I), pp. 185-203

Campos Marín, Ricardo (2003), “Criminalidad y locura en la Restauración. El proceso del cura Galeote (1886-1888)", Frenia, III (2), pp. 111-145. facultativos ${ }^{10}$. Como vemos, pues, el liberalismo y progresismo que se presuponía a la ley por parte de sus mentores quedó pulverizado en su recorrido posterior sirviendo como instrumento de represión para toda la población.

6 MINISTERIO DE JUSTICIA. DECRETO. Gaceta de Madrid, no 332, 28 de noviembre de 1935 , pp. 1715.

7 "En un mitin del Frente Popular, Ruiz Funes hace una crítica severa del segundo bienio", La Voz, 03-02-1936.

8 Luis Jiménez de Asúa, “Por la justicia. La ley de vagos y maleantes, La Libertad, 25-08-1935.

9 LEY DEL 15 DE JULIO por la que se modifican los artículos 2ㅇ y 6응 de la Ley de Vagos y Maleantes de 4 de agosto de 1933. BOE, 17 de julio de 1954, no 198, p. 4862.

10 LEY de 24 de abril de 1958 por la que se crean dos Juzgados especiales y se dictan normas complementarias de la Ley de vagos y Maleantes, de 4 de agosto de 1933. BOE, 25 de abril de 1958, no 99, p. 749; MINISTERIO DE JUSTICIA, Orden de 28 de mayo de 1958 por la que se dictan normas complementarias de la Ley de 24 de abril último sobre Juzgados Especiales de de Vagos y Maleantes. BOE, 4 junio 1958, n 1ㅇ33, p. 1006.
Campos, Ricardo (2007), “¿Psiquiatría para los ciudadanos o psiquiatría para la represión? El problema de la peligrosidad del enfermo mental en España (1920-1936)". En: Campos, Ricardo; Villasante, Olga y Huertas, Rafael (eds.), De la "Edad de plata" al exilio. Construcción y "reconstrucción" de la psiquiatría española, Madrid, Frenia, pp. 15-36.

Campos, Ricardo (2009), "La clasificación de lo difuso: el concepto de "mala vida" en la literatura criminológica de cambio de siglo", Journal of Spanish Cultural Studies, 10, 4, pp. 399-422.

Campos, Ricardo (2012), El caso Morillo: crimen, locura y subjetividad en la España de la Restauración, Madrid, Frenia, CSIC.

Campos Marín, Ricardo; Martínez Pérez, José y Huertas, Rafael (2000), Los ilegales de la naturaleza. Medicina y degeneracionismo en la España de la Restauración (1876-1923), Madrid, CSIC.

Campos, Ricardo y Huertas, Rafael (2013), "Lombroso but not Lombrosians? Criminal anthropology in Spain". En: Kneppe y Ystehede Per Jørgen (Eds.), The Cesare Lombroso Handbook, New York, Routledge, pp. 309-323. 
Casanova, Julián (1997), De la calle al frente. El anarcosindicalismo al frente (1931-1939), Barcelona, Crítica.

Castel, Robert (1980), El orden psiquiátrico. La edad de oro del alienismo, Madrid, La Piqueta.

Cleminson, Richard (2009), "Transnational Discourse on the 'Mala Vida': Male Homosexuality in Madrid, Buenos Aires and BarceIona in the Early Twentieth Century", Journal of Spanish Cultural Studies, 10, 4, december, pp. 461-483.

Cleminson, Richard y Fuentes Peris, Teresa (2009), "Source and Focus of Degeneration. Degeneracy and decline", Journal of Spanish Cultural Studies, 10, 4, december, pp. 385-398.

Conseglieri, Ana y Villasante, Olga (2008), "Un regicida frustrado: la imbecilidad de Otero, según Esquerdo". En: Martínez Pérez, José; Estévez, Juan; Del Cura Mercedes, Víctor y Blas, Luis (coords), La gestión de la locura: conocimiento, prácticas y escenarios (España, siglos XIX-XX), Cuenca, Ediciones de la Universidad de Castilla La Mancha, pp. 283-310.

Dolsa, Luis (1895), Concepto de la degeneración y responsabilidad legal de sus productos mentales. Discurso inaugural del año académico de 1895-96 leído en la Academia y Laboratorio de Ciencias Médicas de Cataluña, Barcelona, Imprenta de Henrich y Cía.

Dörner, Klaus (1969), Bürger und Irre. Zur Sozialgeschichte und issenschaftssoziologie der Psychiatrie, Francfort, Europäische Verlagsanstalt.

Esquerdo, José María (1881), "Locos que no lo parecen”, Revista de Medicina y Cirugía Prácticas, 5, pp. 101-109; 153-159; 211-217; 303-312; 358-365; 402-409.

Fernández, Pura (2001), ¿Loco o delincuente? Novela social contemporánea (1890), Madrid, Celeste.

Foucault, Michel (1990a), Vigilar y Castigar. Nacimiento de la prisión, Madrid, Siglo XXI Editores.

Foucault, Michel (1990b), "La evolución de la noción de individuo peligroso en la psiquiatría legal”. En: Foucault, Michel, La vida de los hombres infames, Madrid, La Piqueta, pp. 260-264.

Foucault, Michel (2001), Los anormales, Madrid, Akal.

Galera, Andrés (1991), Ciencia y delincuencia. El determinismo antropológico en la España del siglo XIX, Sevilla, CSIC.

Gargallo Vaamonde, Luis (2011), El sistema penitenciario de la Segunda República. Antes y después de Victoria Kent (1931-1936), Madrid, Ministerio del Interior-Secretaría General Técnica.

Garofalo, Raffaele (1885), Criminologia. Studio sulle sue cause e sui mezzi di repressione, Torino, Fratelli Bocca.

Garrido Escuin, Victoriano (s.a.), La cárcel o el manicomio. Estudio médico local de la locura. Con un prólogo del Dr. D. José María Esquerdo, Madrid, Casa editorial de don José María Faquineto.

Girón, Alvaro (2002), "Los anarquistas y la criminología de Cesare Lombroso (1890-1914), Frenia. Revista de Historia de la Psiquiatría, II (2), pp. 81-108.
Goldstein, Jan (1987), Console and classify. The French psychiatric profession in the nineteenth century, Cambridge, Cambridge University Press.

González González, Joaquín (1994), La imputabilidad en el Derecho Penal español. Imputabilidad y locura en la España del siglo XIX, Granada, Editorial Comares.

Heredia Urzáiz, Iván (2009), “Control y exclusión social: la ley de vagos y maleantes en el primer franquismo". En: Romero, Carmelo y Sabio, Alberto (coords.), Universo de Micromundos. VI Congreso de Historia Local de Aragón, Zaragoza, Institución «Fernando el Católico» (CSIC), Excma. Diputación de Zaragoza, pp. 109-120.

Heredia Urzaiz, Iván (2006), "La defensa de la sociedad. Uso y abuso de la Ley de Vagos y Maleantes". En: Castillo, Santiago y Oliver, Pedro (eds.), La figuras del desorden. Heterodoxos, proscritos y marginados. Comunicación presentada al V Congreso de Historia Social, Madrid, Siglo XXI.

Huertas, Rafael (2002), Organizar y persuadir: estrategias profesionales y retóricas de legitimación de la medicina mental española (1875-1936), Zaragoza-Madrid, Frenia.

Huertas, Rafael (2004), "Entre la ciencia forense y la legitimación social: En torno al caso Garayo”. En: Álvarez Martínez, José María y Esteban Arnáiz, Ramón (coords), Crimen y locura. IV Jornadas de la sección de Historia, Madrid, Asociación Española de Neuropsiquiatría, pp. 17-33.

Huertas, Rafael (2011), Historia cultural de la psiquiatría. (Re) pensar la locura, Madrid, Los libros de la Catarata.

Huertas, Rafael y Martínez-Pérez, José (1993), “Disease and crime in Spanish positivist psychiatry", History of Psychiatry, 4, pp. 459-481.

Jiménez de Asúa, Luis (1934), Ley de vagos y maleantes: Un ensayo legislativo sobre peligrosidad sin delito, Madrid, Editorial Reus.

Kalifa, Dominique (2005), Crime et culture au XIXe siècle, Paris, Perrin.

Lantéri-Laura, Georges (2000), Ensayo sobre los paradigmas de la psiquiatría moderna, Madrid, Fundación Archivos de Neurobiología, Editorial Triacastela.

Lecha-Marzo, Antonio (1906), "Antropología criminal. Las anomalías de Mateo Morral", Revista de Medicina y Cirugía prácticas, 72, pp. 84-89.

Lecha-Marzo, Antonio (1911a), "Sobre los ligeramente locos", Protocolo Médico-Forense, 13, pp. 99-100.

Lecha-Marzo, Antonio (1911b), "Más sobre los fronterizos", Protocolo Médico-Forense, 13, pp. 101-102.

Lecha-Marzo, Antonio y Piga, Antonio (1915), "La prueba médica del discernimiento", Los Progresos de la Clínica, 5, pp. 352-361.

López, José (1882), "El discurso del doctor Esquerdo", El Criterio Médico, 15-30 de junio, pp. 258-269.

Maristany del Rayo, Luis (1998), "Situación y contexto de La mala vida de Madrid". En: Bernaldo de Quirós, Constancio y Llanas Aguila- 
niedo, José María, La mala vida en Madrid. Estudio psicosociológico con dibujos y fotografías al natural, Huesca, Ed. Justo Broto Salanova-Instituto de Estudios Altoaragoneses, pp. XXXI-LVIII.

Maristany, Luis (1973), El gabinete del doctor Lombroso. (Delincuencia y fin de siglo en España). Barcelona, Anagrama.

Martínez-Pérez, José (1986), "La profilaxis médica del crimen en la obra de Lecha-Marzo", Asclepio, 38 (I), pp. 241-271.

Martínez-Pérez, José (1989), La Medicina Legal en la enseñanza médico-quirúrgica de la España de la llustración, Madrid, Universidad Complutense (Tesis Doctorales, $n$ ㅇ 34/89).

Max-Bembo (1912), La mala vida en Barcelona. Anormalidad, miseria y vicio, Barcelona, Casa Editorial Maucci.

Mira y López, Emilio (1932), Manual de psicología jurídica, Barcelona, Salvat Editores.

Niceforo, Alfredo y Sighele, Scipio (1902), La mala vida en Roma, Madrid, B. Rodríguez Serra.

Ots y Esquerdo, Vicente (1894), La locura ante los tribunales o estudio médico-legal de la irresponsabilidad del loco, Madrid, Centro Editorial de Góngora.

Peset, José Luis (1983), Ciencia y marginación. Sobre negros, locos y criminales, Barcelona, Crítica.

Petit, Carlos (2005), "La célebre causa del crimen de Fuencarral. Proceso penal y opinión pública bajo la Restauración", Anuario de Historia del Derecho Español, 75, pp. 369-411.

Plumed Domingo, José Javier y Rey González, Antonio (2002), “La introducción de las ideas degeneracionistas en la España del siglo XIX. Aspectos conceptuales", Frenia, 2, pp. 31-48.

Prins, Adolphe (1910), La défense sociale et les transformations du droit pénal, Bruxelles, Misch et Thron.

Renneville, Marc (2003), Crime et folie. Deux siècles d'enquêtées médicales et judiciaires, París, Fayard.

Rios-Font, Wadda C. (2005), "El crimen de la calle de San Vicente: Crime Writing and Bourgeois Liberalism in Restoration Spain", MLN, 120 (2), pp. 335-354.
Rodríguez Lafora, Gonzalo (1929), La psiquiatría en el nuevo Código Penal español de 1928, (juicio crítico), Madrid, Reus.

Ruiz Maya, Manuel (1928), "La peligrosidad de los alienados en sus aspectos teórico y práctico". Segunda Reunión Anual de la Asociación Española de Neuropsiquiatras, Madrid, 22, 23, 24 de octubre de 1927, Archivos de Neurobiología, 8, pp. 63-97.

Ruiz Maya, Manuel (1930), “Limite de la peligrosidad en los enfermos mentales y medios para justificar la existencia de las circunstancias que la determinan". En: Asociación Española de Neuropsiquiatras. Tercera Reunión Anual, Bilbao, 22, 23, 24 de septiembre de 1928, Barcelona, Tipografía Santiago Vives.

Sáez Domingo, Agustín (1885), Procesos Célebres Crónicas de Tribunales Españoles. D. Luis Jiménez parricidio de la calle de san Hermenegildo (Madrid). Los bandos de Velilla (Zaragoza), Madrid, Imprenta de la Revista de Legislación.

Saforcada, Manuel y Torras, Oscar (1930), “Comentarios psiquiátricos al nuevo Código Penal español. Cuarta Reunión Anual de la Asociación Española de Neuropsiquiatras, celebrada en Sevilla el 17, 18 y 19 de diciembre de 1929", Archivos de Neurobiología, 10, $443-463$.

Sánchez de Ocaña, Ramón (1887), Proceso Hillairaud: asesinato frustrado del ex mariscal Bazaine. Sumario, juicio oral, sentencia. Proceso de Archidona: recurso de casación, sentencia, Madrid, Imp. Revista General de Legislación, pp. 5-79.

Scull, Andrew (1979), Museums of Madness. The social organization of insanity in nineteenth-century England, London, Allen Lane.

Seoane, María Cruz (1989), Historia del periodismo en España 2. El siglo XIX, Madrid, Alianza Editorial.

Swain, Gladys (1977), Le sujet de la folie. Naissance de la psychiatrie, Toulouse, Privat.

Trinidad Fernández, Pedro (1991), La defensa de la sociedad. Cárcel y delincuencia en España (siglos XVIII-XX), Madrid, Alianza Editorial.

Varela, Julia y Álvarez-Uría, Fernando (Eds.) (1979), El Cura Galeote, asesino del Obispo de Madrid-Alcalá. Proceso Médico-Legal, reconstruido y presentado por Julia Varela y Fernando ÁlvarezUría, Madrid, La Piqueta. 